\title{
Investigation of nematic liquid crystals as surface acoustic wave sensor coatings for discrimination between isomeric aromatic organic vapors
}

\author{
Samuel J. Patrash, Edward T. Zellers * \\ The University of Michigan, School of Public Health, Department of Environmental and Industrial Health, Ann Arbor, \\ MI 48109-2029, USA
}

(Received 23rd August 1993; revised manuscript received 15th October 1993)

\begin{abstract}
The use of thermotropic nematic liquid crystals (LC) as surface acoustic wave (SAW) vapor sensor coatings was investigated. Responses to four pairs of isomeric aromatic organic vapors were measured using two LC coatings and four isotropic polymer coatings. In most cases, the LC coatings showed higher sensitivity toward the more rod-like isomer within a pair due to the anisotropic nature of the deposited LC films. However, the importance of vapor-coating functional-group interactions as mediating factors in the sensor responses was evident in several cases. Incorporation of an LC coating into a four-sensor array improved the discrimination between isomers relative to an array employing only isotropic coatings. A persistent decline in the sensor baseline signal and vapor sensitivity observed over time with both LC coatings could be attributed to evaporative loss and/or changes in the elastic stiffness of the coatings.
\end{abstract}

Key words: Acoustic methods; Sensors; Liquid crystal; Vapor sensor; Surface acoustic wave sensor

\section{Introduction}

The development and characterization of surface coatings continues to be a major focus of research on coated bulk acoustic wave (BAW), surface acoustic wave (SAW) and related piezoelectric vapor sensors [1-4]. The type of coating material employed and the nature of the interactions that occur between the coating and the analyte vapor(s) are key determinants of sensor

\footnotetext{
* Corresponding author.
}

sensitivity and selectivity. With sorptive coatings, such as high-boiling liquids and rubbery polymers, responses vary directly with the extent of equilibrium vapor partitioning into the bulk of the coating. As a result, sensitivity is generally higher for less volatile vapors and for vapors capable of stronger intermolecular interactions with the coating [5]. The coating-vapor interaction strength, in turn, is determined principally by the relative polarities, polarizabilities, and hydrogen-bond donor / acceptor properties of the functional groups present in the interacting species [5-7]. Selective measurement of certain vapors is 
possible by employing an array of sensors overlayed with different functionalized coating materials and then analyzing the response pattern obtained upon vapor exposure [8-10]. However, isomeric or structurally homologous vapors, which have very subtle differences in chemical properties, would not likely to be reliably discriminated from each other using conventional isotropic coating materials.

The use of coating materials capable of differentiating between vapors on the basis of molecular size and shape constitutes an alternative approach to achieving selectivity with BAW or SAW sensors and sensor arrays. Examples include zeolites, cyclodextrins, cyclophanes, calixarenes, and other so-called cavitands that are characterized by the presence of well-defined cavities or pores. Gases and vapors not able to fit within the cavities are not sorbed as strongly. Although a number of preliminary reports have appeared on the behavior of such materials as acoustic-wave sensor coatings [11-15], a systematic study demonstrating size/shape selectivity, while accounting for differences in vapor volatility and functionalgroup interactions with the coating material, has yet to be performed.

In this paper, we present results of an initial investigation of thermotropic nematic liquid crystals (LC) as SAW sensor coatings for discriminating between isomeric and structurally similar organic vapors. LCs are materials that exhibit properties intermediate between those of crystalline solids and isotropic liquids, either over a range of temperatures (as in thermotropic LCs) or due to the presence of solvent (as in lyotropic LCs) [16]. LCs can also be classified by the nature of their structural anisotropy: nematic LCs are characterized by anisotropic domains wherein the molecules are aligned parallel to one another; smectic LCs also exhibit domains of parallel alignment in addition to a well-ordered layered structure; and cholesteric LCs have a spiral anisotropy due to the presence of chiral centers in the constituent molecules.

The LC anisotropy gives rise to solvent properties that differ from those of isotropic liquids [16]. With nematic $\mathrm{LCs}$, for example, solute vapors having more rod-like or planar shapes are often preferentially dissolved. This feature has been exploited in gas chromatography by using LC stationary phases to separate structural isomers (e.g., substituted aromatic compounds) that could not be resolved using standard isotropic stationary phases [17-19].

The first report on the use of LCs as acoustic wave sensor coatings was published by Mierzwinski and Witkiewicz [20]. In that study, responses from an $8-\mathrm{MHz}$ quartz $\mathrm{BAW}$ resonator coated with each of four different nematic LCs were examined upon exposure to several vapors. Included in the set of vapors were the $o$ - and $p$-isomers of diethylbenzene. For most of the vapors, sensor responses varied inversely with vapor boiling point as expected for isotropic coatings. In contrast, the responses for $p$-diethylbenzene were $1-21 \%$ greater than those for $o$-diethylbenzene, even though these isomers have the same boiling point. The differences in responses between these isomers were attributed to the preferential solubility of the more rod-like $p$-isomer in the LC coatings due to the LC anisotropy. Unfortunately, no data were provided on the responses to these isomers using isotropic coatings, and the possibility that the response differences could be accounted for wholly or partly by slight differences in polarity or polarizability between the isomeric vapors was not considered.

In the work described here, SAW sensor responses to several pairs of isomeric aromatic organic vapors are examined using LC coatings and then compared to those obtained using isotropic coatings that span a wide range of structures and polarities. One of our goals was to determine whether partial selectivity for $p$ - or 4-substituted isomers would be observed consistently across different classes of aromatic compounds. In addition, we wanted to assess the relative contributions of structural anisotropy and functionalgroup interactions to SAW sensor responses using LC coatings. Finally, it was of interest to determine whether inclusion of LC coatings in a SAW sensor array would significantly improve the selective identification of isomeric vapors compared to an array employing only isotropic coating materials. 


\subsection{SAW sensor response equations}

Eq. 1 gives an approximate expression for the change in the frequency of a SAW oscillator, $\Delta f_{c}$, upon deposition of a thin, non-conducting, perfectly elastic, isotropic coating film [21,22],

$$
\begin{aligned}
\Delta f_{\mathrm{c}}= & \left(k_{1}+k_{2}\right) f_{\mathrm{o}}^{2} h \rho_{\mathrm{c}}-k_{1} f_{\mathrm{o}}^{2} h 4 \mu[(\lambda+\mu) \\
& /(\lambda+2 \mu)] / V^{2}
\end{aligned}
$$

where $k_{1}$ and $k_{2}$ are substrate constants $(-8.7 \times$ $10^{-8} \mathrm{~m}^{2} / \mathrm{kg} \mathrm{s}$ and $-3.9 \times 10^{-8} \mathrm{~m}^{2} / \mathrm{kg} \mathrm{s}$, respectively, for ST-quartz) [10], $f_{0}$ is the frequency of the uncoated device $(\mathrm{Hz}), h$ is the coating film thickness $(\mathrm{m}), \rho_{\mathrm{c}}$ is the density of the coating material $\left(\mathrm{kg} / \mathrm{m}^{3}\right), V$ is the acoustic wave velocity $(3158 \mathrm{~m} / \mathrm{s}$ for ST-quartz), and $\lambda$ and $\mu$ are, respectively, the Lamé constant and shear modulus of the film $\left(\mathrm{N} / \mathrm{m}^{2}\right)$.

The quantity $h \rho_{\mathrm{c}}$ is equivalent to the mass per unit area of the film and the first term on the right-hand side of Eq. 1 reflects the component of the sensor response arising from the addition (or loss) of mass from the surface of the sensor. Since $k_{1}$ and $k_{2}$ are negative, increases of mass lead to decreases in the output frequency. The second term on the right hand side of Eq. 1 depends primarily on the elastic stiffness or, more specifically, the shear modulus of the coating film, $\mu$. The effective value of $\mu$ is frequency dependent and will therefore increase with the operating frequency of the SAW sensor $[10,23,24]$. For coatings whose shear moduli are in the range of $10^{7}-10^{9} \mathrm{~N} / \mathrm{m}^{2}$, the magnitude of the second term of Eq. 1 will be about $1-10 \%$ of that of the first term. However, since $k_{1}$ is negative, the second term will lead to a positive shift in frequency, counteracting the effect of mass loading. The net value of $\Delta f_{\mathrm{c}}$ will then depend on the relative contributions of the first and second terms of the equation. The observation that $\Delta f_{\mathrm{c}}$ is invariably negative upon coating deposition indicates that the effect of mass loading predominates over that of elastic stiffness.

An equation similar to Eq. 1 can be used to describe the change of frequency upon exposure of a coated SAW sensor to a vapor [24,25]. For sorptive polymer coatings, vapor exposure causes an increase in the coating mass and a decrease in elastic stiffness (i.e., softening). In this case, the second term on the right hand side of Eq. 1 is positive and mass loading, softening, or a combination of the two will cause a negative shift in sensor frequency.

It has been shown, however, that the following simplified equation can describe quite accurately the responses of polymer-coated SAW oscillators to organic vapors $[5,24]$ :

$\Delta f_{\mathrm{v}}=\Delta f_{c} K_{\mathrm{e}} C_{\mathrm{v}} / \rho_{\mathrm{c}}$

where $\Delta f_{v}$ is the frequency shift observed upon exposure to a vapor, $C_{\mathrm{v}}$ is the atmospheric vapor concentration, and $K_{\mathrm{e}}$ is the "effective" partition coefficient. For cases where liquid or rubberyamorphous polymer coatings are used, responses to most vapors are linear over a considerable range of concentration and $K_{\mathrm{e}}$ can be used as a summary measure of sensitivity. Values of $K_{\mathrm{e}}$ are typically higher than the partition coefficients determined from gas chromatographic measurements [5,24] presumably because sensor responses are a function of changes in both the mass and modulus of the coating upon vapor sorption. Thus, while $K_{\mathrm{e}}$ is not a true partition coefficient it nonetheless serves as a useful index with which to compare sensor responses to different vapors.

\section{Experimental}

Two commercially available LC coating materials, referred to as E38 and ZLI-389 (EM Industries, Hawthorn, NY), were investigated. E38 is a proprietary mixture of 4-cyano-4'-n-alkylbiphenyls $\left(\rho=1.005 \mathrm{~g} / \mathrm{cm}^{3}\right)$ that exhibits a stable nematic phase between -9 and $85^{\circ} \mathrm{C}$. ZLI-389 is a proprietary mixture of two types of aromatic esters $\left(\rho=1.077 \mathrm{~g} / \mathrm{cm}^{3}\right)$ with a nematic range between -20 and $60^{\circ} \mathrm{C}$. Aside from their commercial availability, these particular LCs were chosen for study because they have nematic ranges that bracket normal ambient temperatures and, unlike many common LCs, they are chemically stable in the presence of atmospheric oxygen and water vapor. Their basic structures are shown below. 
E38

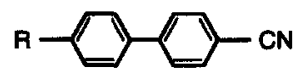

ZLI-389
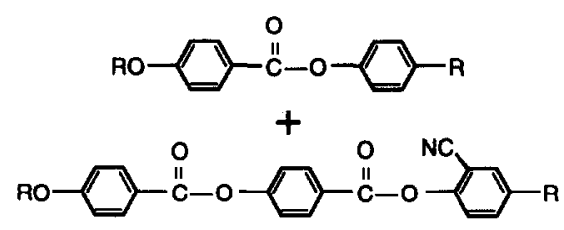

The following isotropic coatings were also examined: poly(bis(cyanoallyl)siloxane) $(\rho=1.001$ $\mathrm{g} / \mathrm{cm}^{3}$, OV-275), poly(methylphenylsiloxane) ( $25 \%$ methyl, $\left.\rho=1.15 \mathrm{~g} / \mathrm{cm}^{3}, \mathrm{OV}-25\right)$, poly(phenyl ether) six-rings $\left(\rho=1.22 \mathrm{~g} / \mathrm{cm}^{3}, \mathrm{PPE}\right)$, Apiezon-L (a branched hydrocarbon grease, $\rho=$ $0.892 \mathrm{~g} / \mathrm{cm}^{3}$, APL) (Anspec, Ann Arbor, MI), and poly(isobutylene) $\left(\rho=0.918 \mathrm{~g} / \mathrm{cm}^{3}\right.$, PIB $)$ (Aldrich, Milwaukee, WI). Solutions of the coatings were prepared in one of several organic solvents and applied to the sensors using an airbrush. For each coating material, the deposition process was monitored via the sensor frequency shift and concluded when $\Delta f_{\mathrm{c}}$ reached approxi- mately $200 \mathrm{kHz}$ (note: the actual quantity measured was the difference frequency between the coated device and an uncoated reference device, and since the frequency of the coated sensor was initially lower than that of the reference sensor, decreases in the coated sensor frequency correspond to increases in the difference frequency).

The solvents examined are listed in Table 1 along with their respective boiling points [26]. All solvents were obtained from Aldrich and were greater than $98 \%$ pure, with the exception of the lutidine (i.e., dimethylpyridine) isomers which were $96 \%$ pure.

A dual 158-MHz delay-line oscillator configuration, consisting of a coated oscillator and a sealed uncoated reference oscillator, was employed. The oscillators and associated circuitry (i.e., mixers, amplifiers, frequency counters, etc.) were obtained from Microsensor Systems, Bowling Green, KY. A few initial measurements were collected with an individual sensor pair while the majority were collected with an array of four sensor pairs. In all cases, difference frequencies were collected at 2-s intervals and logged on a personal computer via an RS-232 interface. Coated sensors were fitted with nickel-plated lids each having vapor inlet and outlet ports. The lids were held in place with clamps and a Teflon ${ }^{\circledR}$ gasket was used to seal the lids to the T-08

Table 1

$K_{\mathrm{e}}$ ratios of rod-like to non-rod-like isomers for all coatings

\begin{tabular}{|c|c|c|c|c|c|c|c|c|}
\hline \multirow[t]{2}{*}{ Vapor pair } & \multirow[t]{2}{*}{ b.p. $\left({ }^{\circ} \mathrm{C}\right)$} & \multicolumn{7}{|c|}{ coating } \\
\hline & & APL & PIB & OV-25 & PPE & OV-275 & ZLI-389 & E-38 \\
\hline$p$-Xylene $/ m$-xylene & $138 / 139$ & 1.04 & 0.97 & 1.04 & 0.97 & 1.08 & 1.05 & 1.16 \\
\hline Standard deviation & & 0.02 & nd & nd & nd & 0.03 & 0.01 & 0.04 \\
\hline$n$ & & 5 & 1 & 1 & 1 & 2 & 3 & 8 \\
\hline 4-Methylstyrene / $\alpha$-methylstyrene & $169 / 163$ & 1.25 & 1.06 & 1.04 & 1.01 & 1.26 & 1.49 & 1.65 \\
\hline Standard deviation & & 0.05 & nd & nd & nd & 0.02 & 0.03 & 0.02 \\
\hline$n$ & & 2 & 1 & 1 & 1 & 2 & 3 & 3 \\
\hline 4-Chlorotoluene /2-chlorotoluene & $162 / 159$ & 1.02 & 0.91 & 0.97 & 1.04 & 1.09 & 1.23 & 1.21 \\
\hline Standard deviation & & 0.02 & nd & nd & nd & 0.10 & 0.04 & 0.03 \\
\hline$n$ & & 2 & 1 & 1 & 1 & 2 & 3 & 2 \\
\hline 2,5-Lutidine /2,4-lutidine & $157 / 159$ & 0.90 & 0.87 & 0.96 & 0.90 & 0.86 & 0.95 & 0.91 \\
\hline Standard deviation & & 0.01 & nd & nd & nd & 0.01 & 0.05 & 0.04 \\
\hline$n$ & & 2 & 1 & 1 & 1 & 2 & 3 & 3 \\
\hline
\end{tabular}

$n=$ Number of determinations, nd $=$ not determined. 
headers on which the sensors were mounted. Sensor temperatures were maintained at $25 \pm$ $0.1^{\circ} \mathrm{C}$ by contacting the lids either with heating tape or with an aluminum block through which thermostatted water was circulated. The temperature was monitored with a thermocouple threaded inside of one of the sensor lids.

Dynamic test atmospheres of each vapor were generated by bubbling nitrogen through the liquid solvent and then diluting with a stream of filtered clean air controlled to $25^{\circ} \mathrm{C}$ and $50 \%$ relative humidity (Model HCS-302 Flow-Humidity-Temperature Controller, Miller-Nelson Research, Carmel Valley, CA). Vapor concentrations were monitored continuously with an infrared gas analyzer (MIRAN 1A, Foxboro, Bridgewood, MA) placed in-line upstream from the sensors. The sensors were connected to the system via stainless steel or Teflon ${ }^{\text {tubing and a }}$ four-port valve was used to expose the sensor(s) alternately to clean air and air containing the test vapor. The flow rates over the sensors were maintained at approximately $0.080 \mathrm{l} / \mathrm{min}$ and were monitored with downstream rotameters.

Each measurement sequence consisted of an initial exposure to clean humidified air to establish a stable baseline frequency followed by duplicate 40 -s exposures to a pre-set concentration of the test vapor. Each vapor exposure was separated by a $40-\mathrm{s}$ exposure to clean air. Responses and recoveries were quite rapid, with stable readings being attained within $10-20 \mathrm{~s}$ of introduction or removal of the vapor stream. Readings from the last $10 \mathrm{~s}$ of each exposure period were averaged and the baseline subtracted to obtain the net sensor response at a given vapor concentration. Each vapor was tested at four different concentrations over a 4-10 fold range. Pairs of isomeric vapors were tested over similar concentration ranges.

\section{Results and discussion}

\subsection{Responses to isomeric vapors}

Sensor responses to the vapors were linear for all coatings over the concentration ranges tested and yielded calibration plots with linear regression correlation coefficients $\left(r^{2}\right)>0.99$. Values of $K_{e}$ were calculated for each vapor/coating combination from the average of the responses at each concentration (Eq. 2). $K_{e}$ for the isotropic coatings ranged from about 2000 to 25000 and calculated limits of detection (LOD) ranged from 10 to $110 \mu \mathrm{g} / 1$ (the LOD is defined here as the concentration producing a signal-to-noise ratio of three, where a noise level of $15 \mathrm{~Hz}$ is assumed). Specific values of $K_{\mathrm{e}}$ and LOD for each isotropic coating/vapor combination have been published elsewhere [5]. For the LC coatings, initial $K_{e}$ values (see below) ranged from 3600 to 21000 and LODs ranged from about 10 to $60 \mu \mathrm{g} / 1$. Typical values of $K_{\mathrm{e}}$ for E38 and ZLI-389 were, respectively, 4380 and 4720 ( $p$-xylene), 3660 and 4440 ( $m$-xylene), 21400 and 21300 (4-methylstyrene), 12900 and 14500 ( $\alpha$-methylstyrene), 10 700 and 12200 (4-chlorotoluene), 9030 and 10 300 (2-chlorotoluene), 12500 and 13400 (2,5lutidine), and 13400 and 14900 (2,4-lutidine). Intercoating reproducibility, as determined from the average $K_{\mathrm{e}}$ for $m$-xylene from fresh replicate coating films $(n=2-5)$, was within the range of $\pm 15 \%$ for the LC coatings.

Since our primary interest is in the relative responses to the various isomers, the average $K_{e}$ value for each rod-like (i.e., $p$ - or 4-substituted) isomer has been divided by that for the corresponding non-rod-like (i.e., $o-, m$-, or $\alpha$-substituted) isomer for each coating (note: for the lutidines, the 2,5-isomer is considered to be more rod-like than the 2,4 isomer). Table 1 presents the ratios of the $K_{\mathrm{e}}$ values for each isomer pair on the isotropic and LC coatings. For sensor responses determined more than once with the same deposited coating film or with different films of that material, the average $K_{\mathrm{e}}$ ratio, standard deviation and number of determinations are provided in Table 1 . The calculation of any particular ratio was always based on isomer responses collected on the same day.

For the xylenes, the $p$-isomer to $m$-isomer response ratios observed with the isotropic coatings ranged from 0.97 to 1.08 , while that for E38 was 1.16 . The ratio for E38 is significantly larger than the highest ratio observed with the isotropic 
coatings as determined by a small-sample $t$-test of the respective average ratios $(p<0.025)$. The ratio for the other LC coating, ZLI-389, was 1.05 which is within the range observed with the isotropic coatings. Although the ratios for the isotropic coatings are all close to one, the fact that three of the five isotropic coatings gave response ratios greater than one belies the fact that the boiling point for the $p$-xylene is slightly lower than that of $m$-xylene. The substantially larger ratio observed for E38 suggests at least some degree of structural anisotropy in the deposited coating film. The failure to observe a larger response ratio with ZLI-389 may be due to the lack of anisotropic alignment in the deposited film, the disruption of alignment upon vapor sorption, and/or the predominance of chemical (i.e., functional group) factors over shape factors in the overall coating-vapor interaction.

For the chlorotoluenes, the 4-isomer to 2-isomer $K_{\mathrm{e}}$ ratios range from 0.91 to 1.09 on the isotropic coatings, whereas both of the LC coatings give ratios of about 1.2 . In this case, a higher response was expected for the higher boiling 4isomer. Statistical comparisons show that the ZLI-389 ratio is significantly larger than that of the highest isotropic-coating ratio $(p<0.10)$, but that the E38 ratio is not $(p>0.10)$ most likely due to the small sample sizes used. The similarity of the ratios for the LC coatings is curious in light of the data for the xylenes: since the chlorine atom is similar in size to the methyl group, the response ratios for the chlorotoluenes with $\mathrm{ZLI}$ 389 were expected to be comparable to those for the xylenes. This provides further evidence of the importance of factors other than shape in affecting responses with this LC coating.

For the methylstyrene isomers the isotropic coatings consistently gave 4 -isomer to $\alpha$-isomer ratios $>1$ (range $=1.01-1.26$ ), accordant with the order of boiling points. Once again, however, the LC coatings gave ratios significantly larger than those for the isotropic coatings: $1.49(p<0.005)$ and $1.65(p<0.005)$ for ZLI-389 and E38, respectively. That the larger ratios observed with the LC coatings are attributable principally to differences in shape between these isomers is supported by photoelectron spectroscopic data showing that in $\alpha$-methylstyrene the ring carbon atoms and pendant ethylene carbon atoms are not coplanar [27], in contrast to styrene (and, presumably 4 -methylstyrene). Thus, the $\alpha$-methylstyrene would not be expected to fit as well into the parallel LC domains.

Finally, for the lutidines the isotropic coatings gave 2,5-isomer to 2,4-isomer response ratios ranging from 0.86 to 0.96 , again, consistent with the slightly higher boiling point of the latter isomer. In this case, however, neither LC coating showed preferential response toward the more rod-like 2,5-lutidine, and the response ratios were similar to those for the isotropic coatings (i.e., ZLI-389 $=0.95$ and E38 $=0.91$ ). Factors related to chemical interactions with these relatively polar isomers apparently predominate completely over shape factors for both of the LC coatings.

\subsection{Responses to other structurally similar vapors}

The preceding results indicate that the LC anisotropy can indeed influence the relative responses to isomeric aromatic vapors due to differences in their shapes, but also that functionalgroup interactions are important mediating factors. To explore this issue further, responses to cyclohexane, cyclohexene and benzene were examined using the same set of coatings as above. These vapors were selected, in part, based on a previous report describing their analysis by gas chromatography using LC stationary phases and several unspecified isotropic stationary phases [17]. The finding that these vapors eluted in the order given above on the LC stationary phases was attributed to the progression in structure from the planar benzene to the non-planar cyclohexane. These results were contrasted to those observed using the isotropic phases, where these vapors eluted in the order of boiling points (i.e., benzene < cyclohexane < cyclohexene).

Table 2 presents normalized values of $K_{\mathrm{e}}$ for these three vapors on each of the coatings examined. To facilitate comparisons between coatings, the three $K_{\mathrm{e}}$ values for a given coating have been normalized by the highest $K_{\mathrm{e}}$ observed. For the non-polar coatings APL and PIB, the order of $K_{\mathrm{e}}$ follows that of the vapor boiling points, as ex- 
Table 2

Normalized $K_{e}$ values for benzene, cyclohexane, and cyclohexene

\begin{tabular}{lllllllll}
\hline Vapor & $\begin{array}{l}\text { b.p. } \\
\text { ('C) }\end{array}$ & \multicolumn{7}{l}{ Normalized $K_{\mathrm{e}}$ value } \\
\cline { 3 - 8 } & & APL & PIB & E38 & OV- & PPE & OV- \\
& & & & & 25 & & 275 \\
\hline Benzene & 80 & 0.69 & 0.75 & 1.00 & 1.00 & 1.00 & 1.00 \\
Cyclohexane & 81 & 0.82 & 0.84 & 0.27 & 0.44 & 0.26 & 0.12 \\
Cyclohexene & 83 & 1.00 & 1.00 & 0.42 & 0.65 & 0.52 & 0.44 \\
\hline
\end{tabular}

pected. For $\mathrm{E} 38, K_{\mathrm{e}}$ increases from cyclohexane to cyclohexene to benzene $\left(K_{\mathrm{e}}=129,199\right.$, and 475 , respectively), consistent with the expectations based on planarity. However, for the more polar coatings OV-25, PPE and OV-275, the order of $K_{\mathrm{e}}$ values also increases with vapor planarity. Furthermore, larger differences in responses are observed between these vapors with OV-275 than with E38 (note: specific values of $K_{e}$ for these vapors on the isotropic coatings can be found in Ref. 5).

Thus shape differences are not necessarily the overriding factors governing the relative sensor responses (and gas chromatographic retention times) with LC materials. Benzene is not only the most planar vapor, but also the most polar and polarizable, followed in order by cyclohexene and cyclohexane. The presence of the cyano and phenyl functionalities in the E38, alone, could account for the greater affinity for benzene than for the other vapors.

\subsection{Aging effects}

Baseline frequencies and responses to $m$ xylene were monitored over time to assess coating stability. Table 3 presents some typical $\Delta f_{\mathrm{c}}$ and $K_{\mathrm{e}}$ values as a function of time for the $\mathrm{LC}$ coatings and two of the isotropic coatings. Data were collected for all four coatings simultaneously with the sensor array. Following an initial 5-day test period, the sensor array was allowed to stand at room temperature with the sensors covered for approximately three months and then a final exposure to $m$-xylene was performed. All $K_{\mathrm{e}}$ values were calculated using the initial value of $\Delta f_{\mathrm{c}}$ obtained directly after deposition of the coatings. This provides a common reference point for comparing the change of $K_{e}$ to the change of $\Delta f_{\mathrm{c}}$.

For OV-275, $\Delta f_{c}$ was essentially constant with time, whereas for both of the LCs $\Delta f_{c}$ declined substantially (63 and $51 \%$ for E38 and ZLI-389, respectively). For $\mathrm{APL}, \Delta f_{\mathrm{c}}$ was quite constant over the initial 5-day period, but was found to have increased slightly (4\%) prior to the final exposure to $m$-xylene. The $m$-xylene $K_{e}$ value for OV-275 did not change significantly, while that for APL increased by about $15 \%$ and those for E38 and ZLI-389 decreased by 46 and 34\%, respectively. Interestingly, the decreases in $\Delta f_{\mathrm{c}}$ for the $\mathrm{LC}$ coatings were consistently larger than the decreases in their $K_{e}$ values for $m$-xylene, and the increase in $\Delta f_{c}$ for APL was consistently smaller than the increase in $K_{\mathrm{e}}$.

The most obvious explanation for the decline in $\Delta f_{\mathrm{c}}$ and $K_{\mathrm{e}}$ for the $\mathrm{LC}$ coatings is evaporation of the coatings from the sensor surface. The fact that the decline of $K_{\mathrm{e}}$ is less than that of $\Delta f_{\mathrm{c}}$ for

Table 3

Values of $\Delta f_{\mathrm{c}}(\mathrm{kHz})$ and $K_{\mathrm{e}}$ for $m$-xylene over time a

\begin{tabular}{lrccllc}
\hline Coating & Day & \multicolumn{1}{c}{$\Delta f_{\mathrm{c}}$} & $\begin{array}{l}\text { \% Change } \\
\text { in } \Delta f_{\mathrm{c}}\end{array}$ & \multicolumn{1}{c}{$K_{\mathrm{c}}$} & $\begin{array}{l}\text { \% Change } \\
\text { in } K_{\mathrm{e}}\end{array}$ \\
\hline E38 & 1 & 206 & - & $3660(110)$ & - \\
& 3 & 171 & -17 & $3190(70)$ & -13 \\
& 4 & 151 & -27 & $2950(130)$ & -19 \\
& 5 & 142 & -31 & $2930(90)$ & -20 \\
& 135 & 75 & -64 & $1940(90)$ & -47 \\
ZLI-389 & 1 & 239 & - & $4430(70)$ & - \\
& 3 & 216 & -10 & $4290(90)$ & -3 \\
& 4 & 207 & -13 & $4200(50)$ & -5 \\
& 5 & 198 & -17 & $4260(60)$ & -4 \\
OV-275 & 135 & 118 & -51 & $2940(90)$ & -34 \\
& 1 & 196 & - & $1890(110)$ & - \\
& 3 & 197 & 1 & $1950(80)$ & 3 \\
& 4 & 196 & 0 & $1960(70)$ & 4 \\
& 5 & 196 & 0 & $2020(60)$ & 7 \\
APL & 135 & 198 & 1 & $1990(90)$ & 5 \\
& 1 & 206 & - & $5120(110)$ & - \\
& 3 & 207 & 0 & $5330(130)$ & 4 \\
& 4 & 208 & 1 & $5350(120)$ & 4 \\
& 5 & 208 & 1 & $5650(120)$ & 10 \\
& 135 & 215 & 4 & $5880(110)$ & 15
\end{tabular}

- Values in parentheses are standard deviations determined from the $K_{e}$ values at four exposure concentrations. 
both LCs may be due to one component of the LC mixture evaporating to a greater extent than the other(s). If $m$-xylene were more soluble in the less volatile $\mathrm{LC}$ mixture component, $K_{\mathrm{e}}$ would not decline as rapidly as $\Delta f_{c}$.

Other aging mechanisms are also possible. According to Eq. 1, a decrease in the coating thickness, $h$, such as would occur upon slow evaporation of the coating, would lead to a proportional decrease in $\Delta f_{c}$, regardless of the relative contributions of the first and second terms of the equation to $\Delta f_{\mathrm{c}}$. Vapor exposure would then yield a proportionally lower $K_{\mathrm{e}}$ value. If, however, the coating evaporation were coupled with an increase in the shear modulus, then $K_{\mathrm{e}}$ might decline to a lesser extent than $\Delta f_{\mathrm{c}}$. Even without any loss of coating, a gradual increase in the shear modulus over time would lead to a decrease in the magnitude of $\Delta f_{\mathrm{c}}$, and most likely some loss in sensitivity.

The increase in $\Delta f_{c}$ for APL can also be explained in terms of modulus changes: a slow relaxation of chain segments in this oligomer could easily account for a decrease in the modulus, and the disproportionate increase in $K_{\mathrm{e}}$ for APL might be due to an increase in vapor sorption capacity accompanying the increased segmental mobility of the coating film.

The data presented in the previous sections indicate that the LC coatings do possess some degree of anisotropy. However, in the absence of an orienting electric field which would align all molecules in the coating in a common direction, deposited LC coating films most likely consist of a series of small domains where the molecules within each domain have a common average alignment but where successive domains are not necessarily aligned with each other [17]. Over time, the degree of orientation of the domains might increase, leading to an increase in the shear modulus of the coating film (note: although the electric field accompanying wave propagation on the SAW device could contribute to such a change, the oscillation of the field is of too high a frequency to cause synchronous "switching" of the LCs [28]). It is known that vapor solubility in the nematic phase of a substance is less than that in the isotropic phase of the same substance due to both entropic and enthalpic factors $[17,19]$. Thus, an increase in average alignment within the film would lead to a reduction in $K_{\mathrm{e}}$.

The plausibility of modulus changes accounting for the observed changes in $\Delta f_{\mathrm{c}}$ can be assessed by using Eq. 1 to estimate the change of modulus required to account for the observed changes in $\Delta f_{\mathrm{c}}$ for the LC coatings under the assumption that the entire shift in $\Delta f_{\mathrm{c}}$ is due to shear modulus changes (note: it is recognized that Eq. 1 is strictly applicable to elastically isotropic coatings, but it should still serve as a useful, if only approximate, model). For E38, for example, the net change in $\Delta f_{c}$ over 135 days was $-63 \%$ or $131 \mathrm{kHz}$ out of the total initial value of $200 \mathrm{MHz}$. If we assume a coating thickness of 60 $\mathrm{nm}$ and a value of 0.84 for the quantity [ $\lambda+$ $\mu) /(\lambda+2 \mu)]$ in Eq. 1 (this quantity is constrained to be between about 0.67 and 1.0), then a modulus change of $6.3 \times 10^{9} \mathrm{~N} / \mathrm{m}^{2}$ would be required, regardless of the initial value of the shear modulus.

Although the actual value of the shear modulus of this LC coating is not known, shear moduli derived for several organic polymers from ultrasonic velocity measurements in the low- $\mathrm{MHz}$ frequency range [23] suggest that a value somewhere in the range of $10^{7}$ to $10^{9} \mathrm{~N} / \mathrm{m}^{2}$ would be expected for the LC coatings at a frequency of $158 \mathrm{MHz}$. Table 4 summarizes the magnitude of the change in $\mu$ required for various possible initial values in this range. For an initial modulus of $10^{7} \mathrm{~N} / \mathrm{m}^{2}, \mu$ must increase by a factor of more than 600 to account for the shift in $\Delta f_{c}$, whereas for an initial value of $10^{9} \mathrm{~N} / \mathrm{m}^{2}$, only about a 7-fold increase is necessary. Thus, one cannot rule out, a priori, the possibility that the

Table 4

Changes in shear modulus, $\mu$, required to account for the baseline drift of the E38-coated sensor for various initial values of $\mu$ (see text)

\begin{tabular}{llc}
\hline $\begin{array}{l}\mu_{\text {initial }} \\
\left(\mathrm{N} / \mathrm{m}^{2}\right)\end{array}$ & $\begin{array}{l}\mu_{\text {final }} \\
\left(\mathrm{N} / \mathrm{m}^{2}\right)\end{array}$ & $\mu_{\text {final }} / \mu_{\text {initial }}$ \\
\hline $10^{9}$ & $7.3 \times 10^{9}$ & 7.3 \\
$10^{8}$ & $6.4 \times 10^{9}$ & 64 \\
$10^{7}$ & $6.3 \times 10^{9}$ & 630 \\
\hline
\end{tabular}


observed decline in $\Delta f_{c}$ is entirely due to modulus changes over time.

Additional insight into this issue was obtained from a separate series of exposures to both $m$ and $p$-xylene performed with the E38 coating over 26 days. Although the sensitivity declined with time, the magnitude of the decline was essentially the same for both vapors. The $K_{\mathrm{e}}$ values obtained with the freshly deposited coating were 4380 and 3660 for $p$ - and $m$-xylene, respectively, yielding a ratio of 1.20 . After 26 days, the respective $K_{e}$ values had declined to 2720 and 2270 , but the ratio remained constant at 1.20 . If the decline in $K_{\mathrm{e}}$ were due to modulus changes associated with an increase in parallel alignment of the LC domains, then one would expect to see an increase in the $p$-xylene to $m$-xylene response ratio. The fact the response ratios did not change suggests that there was no significant change in alignment or modulus. Thus, the observed declines in $\Delta f_{\mathrm{c}}$ and $K_{\mathrm{e}}$ are most likely due to evaporative loss with perhaps a small contribution from modulus effects.

\subsection{Isomer discrimination with a sensor array}

Notwithstanding their instability, it was of interest to determine whether inclusion of LC coatings in an array of SAW sensors would improve the capability for differentiating individual isomers from one another. To this end, a compari-

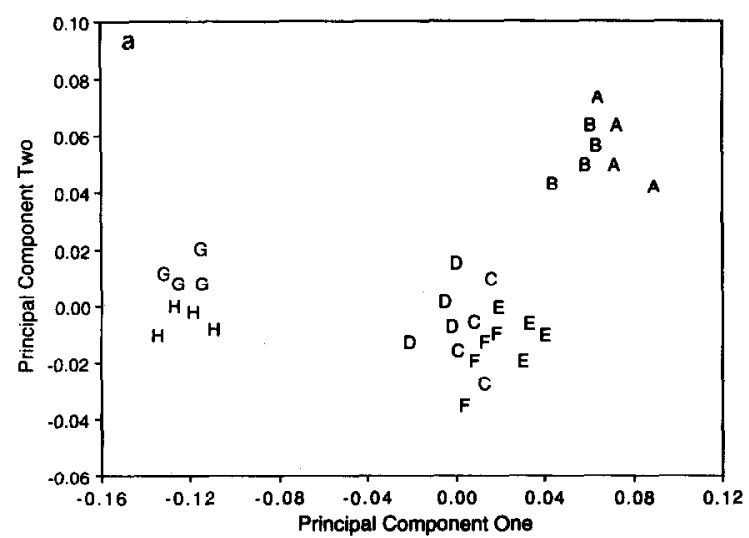

son was made between response patterns obtained from an array of four SAW sensors employing the isotropic coatings OV-275, PIB, OV25 and PPE and an array where one of the isotropic coatings was replaced by the $\mathrm{LC}$ coating E38. The PPE coating was selected for replacement because it was the most similar to E38 in terms of the absolute and relative magnitudes of responses to the test vapors. Thus, replacing this coating minimized any differences in the overall range of polarities spanned by the two arrays.

The performance of each array was first evaluated using a principal components analysis of the response data after pattern normalizing to remove the effect of concentration (i.e., for each test concentration, the individual sensor response was divided by the sum of responses from all four coatings in the array) [29]. Projection of the resulting response vectors onto the plane defined by the first two principal components provides an indication of the degree to which the responses to each vapor differ from one another.

Fig. 1a and $b$ shows the principal components plots for the two arrays being considered. Each point represents the response projection for an individual vapor at a given concentration. For the all-isotropic array, $m$ - and $p$-xylene cluster quite close together while for the array containing E38 the distance between $m$ - and $p$-xylene clusters is greatly increased. A similar improvement in separation is observed between 2- and 4-chloro-

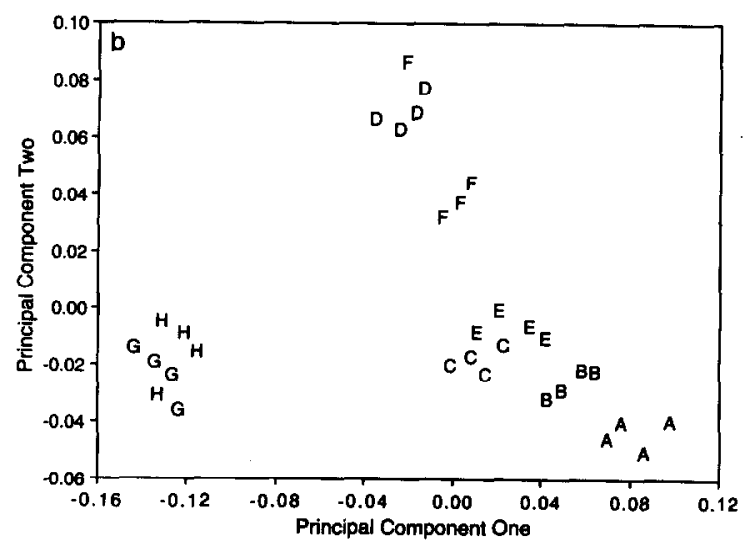

Fig. 1. Principal components analysis projections of response vectors for a four-sensor array with (a) four isotropic coatings and (b) three isotropic coatings and one LC coating (see text). $\mathrm{A}=m$-xylene, $\mathrm{B}=p$-xylene, $\mathrm{C}=\alpha$-methylstyrene, $\mathrm{D}=4$-methylstyrene, $\mathrm{E}=$ 2-chlorotoluene, $\mathrm{F}=$ 4-chlorotoluene, $\mathrm{G}=2$,4-lutidine, $\mathrm{H}=2,5$-lutidine. 
toluene, and an even greater improvement is seen for the isomeric methylstyrene clusters. For the lutidines, where the $K_{e}$ ratios were similar for the isotropic and E38 coatings, no increase in separation is observed with the use of the LC in the array. The overall improvement in isomer discrimination with inclusion of the LC coating is apparent, though it is also clear that the isotropic array will show some discrimination between isomers as well.

A more quantitative comparison of the two arrays was then performed using a disjoint principal components regression classification method [30]. In this method the first principal component determined from the array responses to each of the four test concentrations is used to model each vapor. The model created for each vapor is then used in classification procedures. That is, the response vector obtained upon subsequent exposure to a test vapor is compared successively to the principal component vectors of the previously modeled vapors. The test vapor is then identified on the basis of which principal component vector most closely matches that of its response vector.

In lieu of a separate set of response data, a simulated test set was created from the existing calibration set by superimposing Gaussian error (one standard deviation $=5 \%$ ) on the sensor responses at each concentration and then sampling from the simulated distribution of responses. Each isomer pair was analyzed separately and for each isomer within a pair 40 simulated responses were classified. Error rates were determined from the fraction of incorrect isomer classifications out of the 80 total simulated responses. With the isotropic array error rates of $11,10,19$ and $14 \%$ were obtained for the xylenes, chlorotoluenes, methylstyrenes and lutidines, respectively. As acknowledged above (and as shown in Fig. 1a), the isotropic array is capable of providing some discrimination between the isomers, but the error rates are somewhat higher than one would desire. For the array containing the LC coating there were no misclassifications for the first three pairs of isomers, while for the lutidines the error rate was the same as that for the isotropic array (i.e., 14\%). The latter result was expected since the relative responses to the lutidine isomers for $\mathbf{E 3 8}$ were similar to those for PPE (and the other isotropic coatings). The improvement in classification for the other isomers, however, is significant.

\section{Conclusions}

This brief investigation of LC SAW coatings has yielded several noteworthy results. The importance of the structural anisotropy in as-deposited LC coating films was confirmed. However, it was also shown that the two LC materials investigated here exhibited different behavior and that the ability to discriminate between isomers on the basis of shape alone was not universal. That is, the shape discrimination is mediated by the same types of functional-group interactions that govern responses with isotropic coatings. Nonetheless, inclusion of LC coating materials in a SAW sensor array can enhance the ability to selectively identify certain vapors in the presence of their structural isomers compared to an array containing only isotropic coatings.

The finding that the alkyl cyanobiphenyl LC, E38, gave better discrimination than the aromatic ester LC, ZLI-389, is consistent with previous findings reported by Mierzwinski and Witkiewicz [20] for the one pair of isomers that they examined. It is not clear, however, why the cyanobiphenyl structure generally performs better, i.e., whether it is due to a greater degree of anisotropy or to differences in chemical interaction strengths with the vapors. There is some evidence indicating that cyanobiphenyls show a greater degree of alignment than certain other LC materials under the influence of an electric field [16]. Perhaps they also exhibit a greater degree of spontaneous alignment or are influenced more by interactions with the polar quartz surface of the sensor substrate than are LCs of other structures.

The persistent baseline drifts and losses of sensitivity of sensors coated with the LC materials examined here limit their practical utility. The evidence presented above suggests that evaporative loss of the coating is primarily responsible for these effects, but changes in shear modulus arising from changes in the average alignment of the 
LCs over time may also have contributed. It should be possible to find less volatile nematic LCs that would not have this problem. A number of polymeric LCs with nematic ranges in the ambient temperature range are known $[16,32]$ and would appear worth pursuing in light of the results obtained here.

Certain properties of LC materials, which were not investigated in this study, deserve mention. For example, the application of an orienting electric field as a preconditioning step in order to vary, or optimize, the degree of alignment in the LC coatings may be useful. While this will tend to decrease the average sensitivity to all vapors, it should also increase the selectivity for rod-like or planar molecules. Given the rather high sensitivity exhibited by the LCs used here, some loss in sensitivity could be tolerated in exchange for the improved selectivity expected with more highly oriented coating films. Another potentially useful feature of LCs is their susceptibility to abrupt phase transitions (i.e., from nematic to isotropic) in the presence of high concentrations of vapor phase solutes [16]. The large changes in sorbed mass and coating stiffness accompanying such transitions would lead to large changes in sensor response and might be used to trigger alarms in automated vapor monitoring systems. These issues are currently being investigated.

\section{Acknowledgment}

This work was funded by Grant K01-OHO0077 from the National Institute for Occupational Safety and Health of the Centers for Disease Control.

\section{References}

[1] M.S. Nieuwenhuizen and A. Venema, Sensors and Materials, 5 (1989) 261.

[2] J.J. McCallum, Analyst, 114 (1989) 1173.

[3] M.D. Ward and D.A. Buttry, Science, 249 (1990) 1000.

[4] R.C. Hughes, A.J. Ricco and M.A. Butler, Science, 254 (1991) 74.

[5] S.J. Patrash and E.T. Zellers, Anal. Chem., 65 (1993) 2055.
[6] D.S. Ballantine, Jr., S.L. Rose, J.W. Grate and H. Wohltjen, Anal. Chem., 58 (1986) 3058.

[7] J.W. Grate and M.H. Abraham, Sensors Actuators, B3 (1991) 85.

[8] S.L. Rose-Pehrrson, J.W. Grate, D.S. Ballantine, Jr. and P. Jurs, Anal. Chem., 60 (1988) 2801.

[9] W.P. Carey, K.R. Beebe and B.R. Kowalski, Anal. Chem., 59 (1987) 1529.

[10] H. Wohltjen, D.S. Ballantine, Jr. and N.L. Jarvis in R.W. Murray, R.E. Dessy, W.R. Heineman, J. Janata and W.R. Seitz (Eds.), Chemical Sensors and Microinstrumentation, ACS Symposium Series 403, American Chemical Society, Washington, DC, 1989, pp. 157-175.

[11] T. Bein, K. Brown, G.C. Frye and C.J. Brinker, J. Am. Chem. Soc., 111 (1989) 7640.

[12] C.S.I. Lai, G.J. Moody, J.D.R. Thomas, D.C. Mulligan, J.F. Stoddart and R. Zarzycki, J. Chem. Soc. Perkins Trans. II, (1988) 319.

[13] F.L. Dickert, Th. Bruckdorfer, H. Feigl, A. Haunschild, V. Kuschow, E. Obermeier, W.-E. Bulst, U. Knauer and G. Mages, Sensors Actuators, B13 (1993), 297.

[14] P. Nelli, E. Dalcanale, G. Faglia, G. Sberveglieri and P. Soncini, Sensors Actuators, B13 (1993) 302.

[15] F.L. Dickert and P.A. Bauer, Adv. Mater., 3 (1991) 436.

[16] G. Meier, E. Sackmann and J.G. Grabmaier, Applications of Liquid Crystals, Springer Verlag, Berlin, 1975.

[17] Z. Witkiewicz, J. Chromatogr., 251 (1982) 311.

[18] G.M. Janini, in J.C. Giddings, E. Grushka, J. Cazes and P.R. Brown (Eds.), Advances in Chromatography, Marcel Dekker, New York, 1979, p. 231.

[19] H. Rotzche, Stationary Phases in Gas Chromatography, Elsevier, Amsterdam, 1991, p. 291.

[20] A. Mierzwinski and Z. Witkiewicz, Talanta, 34 (1987) 865.

[21] H. Wohltjen, Sensors Actuators, 5 (1984) 307.

[22] B.A. Auld, Acoustic Fields and Waves in Solids, Vol. 2, Wiley, New York, 1973, p. 271.

[23] E.T. Zellers, R.M. White and S.W. Wenzel, Sensors Actuators, 14 (1988) 35

[24] J.W. Grate, M. Klusty, R.A. McGill, M.H. Abraham, G. Whiting and J. Andonian-Haftvan, Anal. Chem., 64 (1992) 610.

[25] D.S. Ballantine, Jr., Anal. Chem., 64 (1992) 3069.

[26] R.C. Weast, D.R. Lide, M.J. Astle and W.H. Beyer (Eds.), CRC Handbook of Chemistry and Physics, 70th edn., CRC Press, Boca Raton, FL, 1989.

[27] J.P. Maier and D.W. Turner, J. Chem. Soc. Faraday Trans. II, 69 (1973) 196.

[28] T. Ikeda, T. Sasaki and K. Ichimura, Nature, 361 (1993) 428.

[29] D.L. Massart and L. Kaufman, The Interpretation of Analytical Chemical Data by the Use of Cluster Analysis, Wiley, New York, 1983.

[30] E.T. Zellers, T.S. Pan, S.J. Patrash, M. Han and S.A. Batterman, Sensors Actuators, B12 (1993) 123.

[31] S. Wold, Pattern Recognition, 8 (1978) 127.

[32] G.W. Gray, J.S. Hill and D. Lacey, Mol. Cryst. Liq. Cryst. Lett., 7 (1990) 47. 